\title{
Perioperative goal-directed therapy: what's the best study design to investigate its impact on patient outcome?
}

\author{
Bernd Saugel $^{1}$ - Alexandre Joosten ${ }^{2,3} \cdot$ Thomas W. L. Scheeren $^{4}$
}

Received: 11 August 2018 / Accepted: 15 August 2018 / Published online: 23 August 2018

(c) Springer Nature B.V. 2018

The impact of anesthetic management on short and longterm postoperative outcomes is a subject of current research in anesthesiology [1]. There is increasing evidence that certain aspects of anesthetic management-including perioperative goal-directed blood flow and fluid optimization [2, $3]$ and monitoring of the depth of anesthesia [4, 5]—can have a beneficial impact on postoperative complication rates.

Lima et al. [6] need to be commended for their study investigating the effects of implementing a multimodal "hemodynamic and depth of anesthesia treatment protocol" on patient outcome after major open abdominal cancer surgery [6]. The authors demonstrated that in patients treated after the implementation of the treatment protocol (which included mean arterial pressure, stroke volume variation, cardiac index, central venous oxygen saturation, and bispectral index as targets) the incidence of postoperative delirium was significantly lower and the median hospital length of stay was significantly shorter compared to patients having the same kind of surgery prior to protocol implementation [6].

Bernd Saugel

bernd.saugel@gmx.de; b.saugel@uke.de

Alexandre Joosten

joosten-alexandre@hotmail.com

Thomas W. L. Scheeren

t.w.1.scheeren@umcg.nl

1 Department of Anesthesiology, Center of Anesthesiology and Intensive Care Medicine, University Medical Center Hamburg-Eppendorf, Martinistrasse 52, 20246 Hamburg, Germany

2 Department of Anesthesiology, CUB Erasme University Hospital, Université Libre de Bruxelles, Brussels, Belgium

3 Department of Anesthesia and Intensive Care Medicine, Hôpital Bicêtre Université Paris-Sud, Le Kremlin-Bicêtre, France

4 Department of Anesthesiology, University of Groningen, University Medical Center Groningen, Hanzeplein 1, 9713 GZ Groningen, The Netherlands
The study adds another piece to the puzzle of how perioperative anesthetic management can improve postoperative patient outcome. The study also prompts a discussion about different types of studies in anesthesiological research and triggers the question: What is the best study design to investigate a potentially beneficial impact of perioperative goaldirected therapy on patient outcome?

There is no definite answer to this question because one perfect study design does not exist and the optimal study design depends on the specific research question being asked. Perioperative care is characterized by multidisciplinary teamwork, and postoperative outcomes have various pathophysiologic mechanisms and complex multifactorial etiologies. Therefore, expecting that it is simple to design a study that provides generalizable evidence that a single specific intervention can change a single specific postoperative outcome is unrealistic.

Rather, it takes multiple steps and differently designed studies to progress from hypothesizing that a certain intervention may have a beneficial impact on patient outcome to eventually demonstrating that it works in daily clinical practice. As a first step, animal studies and small observational "proof of concept" studies in humans may be necessary to show that a certain intervention can have an effect on certain physiologic and biologic variables or outcomes. Further explanatory studies may then be performed to describe the details of the therapeutic intervention (dosing, timing, etc.). Feasibility studies might also be necessary to demonstrate that the therapeutic intervention and measurement systems needed to guide the intervention can be safely and precisely used in a clinical setting. Finally, the therapeutic intervention might be tested in a randomized controlled trial (Fig. 1). Smaller exploratory monocentric randomized controlled trials (with complex treatment algorithms, strict inclusion and exclusion criteria, and optimal conditions for the study intervention to work) enable the efficacy of an intervention to be tested to answer the question "Can this intervention work?" [7]. Large pragmatic multicenter randomized controlled 
Randomized controlled trial

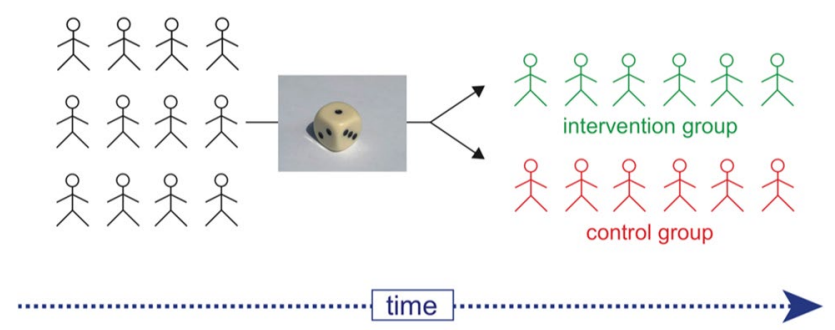

Uncontrolled before-and-after study

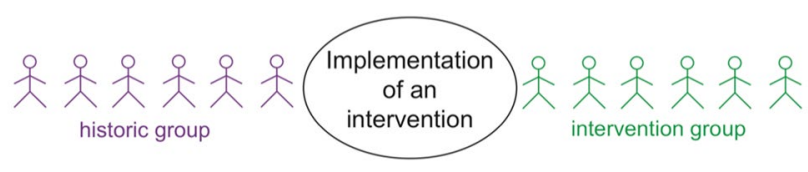

Fig. 1 Schematic illustration of the study design of randomized controlled trials and before-and-after studies

trials (with less complicated treatment algorithms, relatively broad inclusion and exclusion criteria, a well-defined clinical endpoint, and real-world clinical conditions) can ultimately be used to demonstrate the clinical effectiveness and practical feasibility of an intervention in usual care ("Does this intervention work?") [7]. However, a randomized controlled trial is never solely exploratory or pragmatic, but rather can have different degrees of exploratory and pragmatic components [8]. According to the principles of evidence-based medicine high-quality randomized controlled trials and systematic reviews and meta-analysis of these are supposed to provide the highest quality evidence $[9,10]$. However, high-quality pragmatic randomized controlled trials are missing for many anesthesiology treatment strategies. In addition, as discussed previously, in heterogeneous patient populations-such as "surgical patients"-with complex multifactorial outcomes, randomized controlled trials and their meta-analyses have their own limitations and need to be meticulously designed [7, 11-13].

Instead of performing a randomized controlled trial, Lima et al. [6] chose an uncontrolled before-and-after study design [14] (Fig. 1) comparing two periods, the one before (March to August 2013) and that after (March to August 2014) implementation of their treatment protocol.

Before-and-after studies are observational quasi-experimental effectiveness studies and compare the periods before and after the implementation of any new diagnostic or therapeutic strategy, e.g., a therapeutic intervention, treatment protocol, assessment tool, safety initiative, quality improvement project, guideline, or educational program [14]. Because they are simpler to perform, require fewer resources, and bear less ethical concerns than randomized controlled trials, before-and-after studies are frequently used in perioperative research [14], and have been performed to show that perioperative goal-directed therapy can improve patient outcome in high-risk abdominal surgery patients [15-19].

Before-and-after studies can also be used to assess the effectiveness of a newly implemented intervention in comparison with a historical group in a specific setting (institution, hospital, certain operating rooms). Therefore, beforeand-after studies are supposed to enable the impact of an implemented treatment protocol on patient outcome to be assessed under "real-life conditions". To reflect real-life conditions, however, medical caregivers should be blinded to the fact that the implementation of a new treatment is accompanied by a clinical study [14]. This includes that no research coordinators or study nurses should be present during patient treatment, which may impair data quality in general and makes it particularly challenging to guarantee and systematically record the compliance to the protocol. In addition, if possible, researchers assessing the outcome data should be blinded to the study period the patient data were recorded to avoid "observer bias" and should use the same outcome definitions for control and intervention group patients to avoid "measurement bias" [14]. Because the intervention group and the historical group are treated at different time points, before-and-after studies, by definition, are not randomized and thus prone to be confounded by any change in patient management that is not related to the study intervention but occurs before or during its implementation ("history bias") [14]. For example, we may imagine that the standard of care might simply be influenced by changes in the expertise of the medical personnel between the historical period and the intervention period. Moreover, landmark studies or new guidelines suggesting a change in patient management published after the historical period but before or during the intervention period might induce a "learning contamination bias". Both history and learning contamination bias are less likely to occur when the time span between the historical and intervention period is short. To avoid "selection bias" all eligible patients should be treated with the intervention during the intervention period [14].

In summary, before-and-after studies are used in anesthesiology to evaluate the effectiveness of perioperative goal-directed therapy on patient outcome [6, 15-19]. A before-and-after study can give valuable insights into the feasibility and effectiveness of an intervention under real-life clinical conditions and, therefore, can complement evidence from randomized controlled trials. Before-and-after studies, however, are prone to several types of bias and can easily be confounded by factors that are difficult to account for. A profound understanding of advantages and inherent limitations of before-and-after studies is, therefore, key for their conception, analysis, and interpretation, especially regarding definite conclusions about a causal relationship between a 
certain intervention and a certain clinical outcome. To eventually demonstrate that perioperative goal-directed therapy based on pathophysiologic rationale actually improves patient outcome in real-life daily clinical practice we should combine the evidence from complementary studies with different study designs, including both randomized controlled trials and before-and-after studies.

\section{Compliance with ethical standards}

Conflict of interest BS collaborates with Pulsion Medical Systems SE (Feldkirchen, Germany) as a member of the medical advisory board and received honoraria for giving lectures and refunds of travel expenses from Pulsion Medical Systems SE. BS received research support from Edwards Lifesciences (Irvine, CA, USA). BS received institutional research grants, unrestricted research grants, and refunds of travel expenses from Tensys Medical Inc. (San Diego, CA, USA.) BS received honoraria for giving lectures and refunds of travel expenses from CNSystems Medizintechnik AG (Graz, Austria). AJ is consultant for Edwards Lifesciences. TWLS received honoraria from Edwards Lifesciences and Masimo Inc. (Irvine, CA, USA) for consulting and for giving lectures. TWLS received honoraria from Pulsion Medical Systems SE for giving lectures. TWLS is associate editor of the Journal of Clinical Monitoring and Computing but had no role in the handling of this manuscript.

\section{References}

1. Sessler DI. Long-term consequences of anesthetic management. Anesthesiology. 2009;111:1-4. https://doi.org/10.1097/ ALN.0b013e3181a913e1.

2. Pearse RM, Harrison DA, MacDonald N, et al. Effect of a perioperative, cardiac output-guided hemodynamic therapy algorithm on outcomes following major gastrointestinal surgery: a randomized clinical trial and systematic review. JAMA. 2014;311:2181-90. https://doi.org/10.1001/jama.2014.5305.

3. Chong MA, Wang Y, Berbenetz NM, McConachie I. Does goaldirected haemodynamic and fluid therapy improve peri-operative outcomes?: a systematic review and meta-analysis. Eur J Anaesthesiol. 2018;35:469-83. https://doi.org/10.1097/eja.0000000000 000778.

4. MacKenzie KK, Britt-Spells AM, Sands LP, Leung JM. Processed electroencephalogram monitoring and postoperative delirium: a systematic review and meta-analysis. Anesthesiology. 2018;129:417-27. https://doi.org/10.1097/aln.000000000000232 3.

5. Scheeren TWL, Kuizenga MH, Maurer H, Struys M, Heringlake $\mathrm{M}$. Electroencephalography and brain oxygenation monitoring in the perioperative period. Anesth Analg. 2018. https://doi. org/10.1213/ane.0000000000002812.
6. Lima MF, Mondadori LA, Chibana AY, Gilio DB, Giroud Joaquim EH, Michard F. Outcome impact of hemodynamic and depth of anesthesia monitoring during major cancer surgery: a before-after study. J Clin Monit Comput. 2018. https://doi.org/10.1007/s1087 7-018-0190-8.

7. Yeung J, Gillies MA, Pearse RM. Pragmatic trials in peri-operative medicine: why, when and how? Anaesthesia. 2018;73:803-7. https://doi.org/10.1111/anae.14252.

8. Thorpe KE, Zwarenstein M, Oxman AD, et al. A pragmaticexplanatory continuum indicator summary (PRECIS): a tool to help trial designers. J Clin Epidemiol. 2009;62:464-75. https:// doi.org/10.1016/j.jclinepi.2008.12.011.

9. Evidence-Based Medicine Working G. Evidence-based medicine. A new approach to teaching the practice of medicine. JAMA. 1992;268:2420-5.

10. Sackett DL, Rosenberg WM, Gray JA, Haynes RB, Richardson WS. Evidence based medicine: what it is and what it isn't. BMJ. 1996;312:71-2.

11. Joshi GP, Alexander JC, Kehlet H. Large pragmatic randomised controlled trials in peri-operative decision making: are they really the gold standard? Anaesthesia. 2018;73:799-803. https://doi. org/10.1111/anae.14238.

12. Saugel B, Malbrain ML, Perel A. Hemodynamic monitoring in the era of evidence-based medicine. Crit Care. 2016;20:401. https ://doi.org/10.1186/s13054-016-1534-8.

13. Deaton A, Cartwright N. Understanding and misunderstanding randomized controlled trials. Soc Sci Med. 2018;210:2-21. https ://doi.org/10.1016/j.socscimed.2017.12.005.

14. Ho AMH, Phelan R, Mizubuti GB, et al. Bias in before-after studies: narrative overview for anesthesiologists. Anesth Analg. 2018;126:1755-62. https://doi.org/10.1213/ane.0000000000 002705.

15. Kuper M, Gold SJ, Callow C, et al. Intraoperative fluid management guided by oesophageal Doppler monitoring. BMJ. 2011;342:d3016. https://doi.org/10.1136/bmj.d3016.

16. Cannesson M, Ramsingh D, Rinehart J, et al. Perioperative goaldirected therapy and postoperative outcomes in patients undergoing high-risk abdominal surgery: a historical-prospective, comparative effectiveness study. Crit Care. 2015;19:261. https://doi. org/10.1186/s13054-015-0945-2.

17. Habicher M, Balzer F, Mezger V, et al. Implementation of goaldirected fluid therapy during hip revision arthroplasty: a matched cohort study. Perioper Med. 2016;5:31. https://doi.org/10.1186/ s13741-016-0056-x.

18. Malbouisson LMS, Silva JM Jr, Carmona MJC, et al. A pragmatic multi-center trial of goal-directed fluid management based on pulse pressure variation monitoring during high-risk surgery. BMC Anesthesiol. 2017;17:70. https://doi.org/10.1186/s1287 1-017-0356-9.

19. Joosten A, Coeckelenbergh S, Delaporte A, et al. Implementation of closed-loop-assisted intra-operative goal-directed fluid therapy during major abdominal surgery: a case-control study with propensity matching. Eur J Anaesthesiol. 2018;35:650-8. https://doi. org/10.1097/eja.0000000000000827. 